Results In the first 6 months of the 22q11DS Clinic 17 children were assessed. This attendance rate was $94 \%$ and $29 \%$ of this group had their appointment co-ordinated with another specialist on the same day. Overall, 46 children have been seen to date. Following their first assessment, in accordance to the guidelines and clinical need, overall $82 \%$ required surveillance investigations, including: blood testing $(66 \%$ of children), renal ultrasound (30\%) and X-Ray spine (9\%). Specialist referrals were needed in $73 \%$ of children, to a variety of services, most commonly to mental health (48\%), dental (20\%), cardiology (18\%), immunology (14\%), cleft team (14\%), ophthalmology (14\%), audiology (9\%), endocrine $(7 \%)$ and orthopaedics $(7 \%)$.

Conclusion We have identified multiple areas of unmet need with reference to best practice guidance in this dedicated clinic. It is hoped that we can improve care co-ordination further by engaging other specialists to run clinics on the same day, appoint a nurse specialist and adopt a clear care pathway, tailored to the Irish healthcare system using a life course approach to ensure the regular monitoring and anticipation of issues and early intervention that helps in maintaining health, well-being and quality of life.

\section{GP108 NATIONAL PEWS IMPLEMENTATION IN IRELAND; OUTLINING THE EXPERIENCE OF IMPLEMENTING A MANDATED PAEDIATRIC PATIENT SAFETY IMPROVEMENT INITIATIVE}

${ }^{1}$ Rachel MacDonell ${ }^{*}$, ${ }^{2}$ Siobhan Horkan, ${ }^{3} J$ ohn Fitzsimons, ${ }^{3}$ Alf Nicholson. ${ }^{1} R C P I$, Dublin, Ireland; ${ }^{2}$ Portiuncula Hospital, Ballinasloe, Ireland; ${ }^{3}$ Children's University Hospital, Dublin, Ireland

\subsection{6/archdischild-2019-epa.173}

Background The Irish Paediatric Early Warning System (IPEWS) and associated National Clinical Guideline No.12 was developed in response to a ministerial mandate as a funded workstream of the National Clinical Programme for Paediatrics. I-PEWS is a multifaceted approach to improving patient safety and clinical outcomes, based upon the implementation of several complementary interventions, including 5 age-specific paediatric observation charts incorporating a PEWS scoring tool and escalation guide, promotion of effective communication using the national standard (ISBAR communication tool for patient deterioration), timely nursing and medical input, and clear documentation of management plans.

Objectives I-PEWS was developed to improve prevention, recognition and response to children at risk of inpatient clinical deterioration in Ireland. For a defined period, national implementation was overseen by a Working Group of stakeholders and supported by a National Coordinator.

Implementation strategy Over a three-year period, we piloted and refined the PEWS charts and associated education resources and facilitated national implementation of the Irish PEWS in 29 public hospitals. We developed a centrally-delivered, standardised training programme to establish hospital-level PEWS Trainers. Locally nominated PEWS Leads and Trainers were supported by regular communications, site visits and remote support culminating in a paediatric patient safety celebration day to mark the conclusion of the Working Group and Coordinator involvement. National Key Performance Indicators (KPI) were developed to demonstrate adherence to National Clinical Guideline recommendations by the local governance bodies to include development of locally applicable continuous education standards and regular audit to promote frontline ownership of the change.

Results Implementation of PEWS was challenging due to the number of sites involved and the different specialties, resources and levels of engagement within each. The four quarterly KPI reports from 2018 demonstrate inconsistency in implementation and embedding of PEWS.

21 of $29(72 \%)$ hospitals overall report full compliance with the standards set out in the KPI suite. Issues include (ranges indicate variance in reporting across quarters): no local governance group (33\%) no provision for continuous education programme for nurses (1-4 hospitals) or doctors (3-5 hospitals) all admitted children are not monitored using PEWS (1-2 hospitals) audit practices are not as recommended (1-3 hospitals) outcome data is not being collected (4-6 hospitals)

Learning Implementing a national QI initiative is complex. The flexibility for locally relevant adaptations is essential for applicability and buy-in. The KPI data demonstrates a deficit in implementation standards and should be addressed by the Irish health service.

\section{GP109 DOCUMENTATION OF NEUROLOGICAL EXAMINATION WHEN DETERMINING ELIGIBILITY FOR THERAPEUTIC HYPOTHERMIA}

DJ Foley*, PM Filan, BH Walsh. Dept of Neonatology, CUMH, Cork City, Ireland

\subsection{6/archdischild-2019-epa.174}

Introduction Neonatal encephalopathy (NE) is a clinical syndrome characterized by disturbed neurologic function. Severity of NE is based upon standardised neurological examination. In determining eligibility for therapeutic hypothermia (TH), both national and international guidelines require this standardised assessment be performed in the first six hours of life. The appropriate documentation of this early assessment is essential to ensure appropriate management is provided.

Aim To assess if the grade of encephalopathy, and/or a complete neurological exam, was documented among infants admitted with NE when determining eligibility for $\mathrm{TH}$.

Methods A retrospective chart review of infants admitted with NE over a one year period was performed. Approval to conduct this audit was obtained from the local clinical research and ethics committee. The documentation of all assessments conducted following admission was reviewed; data points collected included if a grade of encephalopathy was recorded, and details of any neurological exam documented. The results were presented to staff and trainees, in tandem with educational sessions on performing and documenting a standardised exam. A planned re-audit will be conducted after a 6 month period.

Results Thirty-one patients were admitted with NE over a 12 month period (November 2017 to October 2018, inclusive). Of these infants, twenty-four received $\mathrm{TH}$, and seven did not.

During the first six hours of life, an overall grade of encephalopathy was documented in only nine (29\%) infants admitted with NE. The majority of infants, 28 (90\%), did have at least part of a neurological exam documented during this time, however three infants had none documented. Among 